\title{
聚合离子液体在催化转化果糖制备乳酸中的应用
}

\author{
汪亚美 ${ }^{a}$ 王新承 ${ }^{a}$ 李顺杰 ${ }^{a}$ 黄崇品 $*, a, b$ 宋彦䂞 ${ }^{a}$ 陈标华 $a$ \\ ( ${ }^{a}$ 北京化工大学化工资源有效利用国家重点实验室 北京 100029) \\ ( ${ }^{b}$ 北京化工大学常州先进材料研究院 常州 213000)
}

\begin{abstract}
摘要 化学法分解糖类制备乳酸大都需要苛刻的反应条件, 如高浓度强碱、较高的反应温度等. 介绍了一种以聚合离 子液体 ([IMEP]Cl)为催化剂, 采用水热法直接催化转化果糖制备乳酸的方法, 并对聚合离子液体类型、碱类型、反应温 度、 $[\mathrm{IMEP}] \mathrm{Cl}$ 与 $\mathrm{NaOH}$ 相对量以及底物浓度等实验条件进行了优化. 结果表明, 在 $100{ }^{\circ} \mathrm{C}$ 条件下, 底物浓度为 25 $\mathrm{mmol} / \mathrm{L}$ 时, 反应 $30 \mathrm{~min}$ 乳酸达到最高收率 $65 \%$, [IMEP]Cl 与 $\mathrm{NaOH}$ 浓度分别为 100 和 $50 \mathrm{mmol} / \mathrm{L}$. 该方法有效降低了 水热法合成乳酸的反应温度和碱浓度, 并且[IMEP]Cl 经过 5 次循环利用仍有较好的催化效果, 乳酸收率仍保持在 $55 \%$ 以上. 对该体系催化转化果糖制备乳酸的反应机理进行了初步的推测. 表明以聚合离子液体为催化剂催化转化碳水化 合物制备乳酸有着较好的前景.
\end{abstract}

关键词 聚合离子液体; 果糖; 乳酸

\section{Application of Polymeric Ionic Liquids in the Catalytic Conversion of Fructose to Lactic Acid}

\author{
Wang, Yamei $^{a}$ \\ Wang, Xincheng ${ }^{a}$ \\ Li, Shunjie ${ }^{a}$ \\ Huang, Chongpin*,a,b \\ Song, Yanlei ${ }^{a}$ \\ Chen, Biaohua ${ }^{a}$ \\ ( ${ }^{a}$ State Key Laboratory of Chemical Resource Engineering, Beijing University of Chemical Technology, Beijing 100029) \\ ( ${ }^{b}$ Changzhou Research Institute of Advanced Materials, Beijing University of Chemical Technology, Changzhou 213000)
}

\begin{abstract}
Preparation of lactic acid via chemical transformation of carbohydrates requires rigorous reaction conditions, such as high concentrations of alkali, high temperature, etc. This paper reports the use of polymeric ionic liquids ([IMEP]Cl) as catalyst for the hydrothermal conversion of fructose to lactic acid; the main variables of the reaction, temperature, substrate concentration, as well as the relative amount of [IMEP]Cl and $\mathrm{NaOH}$ were optimized. Results showed that, at $100{ }^{\circ} \mathrm{C}$, substrate concentration of $25 \mathrm{mmol} / \mathrm{L}$, reaction time of $30 \mathrm{~min}$, the highest yield of lactic acid was $65 \%$ with [IMEP] $\mathrm{Cl}$ and $\mathrm{NaOH}$ concentrations of 100 and $50 \mathrm{mmol} / \mathrm{L}$ respectively. This method reduces the reaction temperature and alkali concentration compared to traditional hydrothermal method, additionally it was found that [IMEP]Cl can be used during 5 catalytic cycles without losing its catalytic effect with a lactic acid yield of $55 \%$ in the 5 th reuse cycle. This demonstrates that the use of polymer ionic liquids as catalysts has a better prospect for future developments. A possible reaction mechanism has been proposed, but further investigation is required.
\end{abstract}

Keywords polymeric ionic liquids; fructose; lactic acid

近年来, 环境恶化、能源短缺等问题日益严重, 开 发洁净、可再生的资源成为当前发展的迫切需要 ${ }^{[1 \sim 3]}$. 生物质是植物细胞光合作用的产物, 包括除化石燃料外 的所有来源于动植物、能再生的有机物质 ${ }^{[4]}$. 生物质能 源作为唯一可再生的碳资源, 具有资源丰富、分布面广、
用途广泛、可以转换成多种二次能源等不同于其它可再 生能源的特点, 因而越来越受到人们的青睐 ${ }^{[4,5]}$. 生物质 的开发利用发展迅速, 以生物质为原料已经得到了各种 平台化合物和燃料 ${ }^{[6 ~ 9]}$. 碳水化合物是生物质的主要组 成成分，约占其总量的 $75 \%$. 乳酸作为其下游产品之一,

* E-mail: huangcp@mail.buct.edu.cn

Received August 28, 2014; revised September 26, 2014; published online October 30, 2014.

Project supported by the National Natural Science Foundation of China (No. 21476021) and the National Basic Research Program of China (No. 2010CB226902).

国家自然科学基金(No. 21476021)和国家重点基础研究发展计划(No. 2010CB226902)资助项目. 
化学名为 2-羟基丙酸, 是世界公认的三大有机酸之

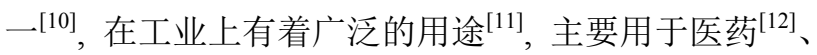
化妆品 ${ }^{[13]}$ 、制药 ${ }^{[14]}$ 以及食品 ${ }^{[15]}$ 等领域, 其高分子聚合物 (聚乳酸)可以进行生物降解, 以其为原料制造可生物降 解性材料有广阔的应用和发展前景 ${ }^{[11]}$.

传统的乳酸制备方法是发酵法, 其生产过程存在菌 种的发酵条件严格、效率低、成本高、产品分离困难、 工艺步骤多等缺点 ${ }^{[16]}$. 因此, 化学方法制备乳酸逐渐成 为当前研究的热点. 化学合成法以乳腈法 ${ }^{[17]}$ 为主, 但是 乳腈法的原料氢氰酸有剧毒, 生产过程会对环境造成较 大污染. Aida 等 ${ }^{[18]}$ 的相关研究表明在没有催化剂存在 时, 葡萄糖在水热条件下 $\left(400{ }^{\circ} \mathrm{C}, 80 \mathrm{MPa}\right)$ 可以分解得到 $6.5 \%$ 乳酸. 以生物质为原料加入适宜的催化剂直接制 备乳酸可以显著降低乳酸的生产成本, 满足绿色化学的 要求. 21 世纪初以强碱为催化剂制备乳酸的研究就已有 报道. Onda 等 ${ }^{[19,20]}$ 报道了以 20 倍(物质的量比)葡萄糖用 量的 $\mathrm{NaOH}$ 为催化剂催化葡萄糖转化为乳酸, 该体系中 $\mathrm{NaOH}$ 用量过大. Yan 等 ${ }^{[21]}$ 发现, $300{ }^{\circ} \mathrm{C}$ 时, 在水溶液体 系中加入 $2.5 \mathrm{~mol} / \mathrm{L} \mathrm{NaOH}$ 或 $0.32 \mathrm{~mol} / \mathrm{L} \mathrm{Ca}(\mathrm{OH})_{2}$ 催化 葡萄糖转化为乳酸, 乳酸的最高收率分别为 $27 \%$ 和 $20 \%$, 该研究虽然降低了碱的用量, 但对反应设备的要 求较高, 并且乳酸收率不高. Zhang 等 ${ }^{[22]}$ 发现, 在 $\mathrm{NaOH}$ 溶液中分别加入 $\mathrm{Zn}, \mathrm{Ni}, \mathrm{C}$ 催化剂, $300{ }^{\circ} \mathrm{C}$ 时, 催化纤维 素制备乳酸, 其最高收率为 $42 \%$. 近年来以无机盐催化 生物质制备乳酸也取得了一定的进展. 以 $\mathrm{ZnSO}_{4}$, $\mathrm{AlCl}_{3} \cdot 6 \mathrm{H}_{2} \mathrm{O}, \mathrm{SnCl}_{2}$ 和 $\mathrm{SnCl}_{4} \cdot 5 \mathrm{H}_{2} \mathrm{O}$ 等金属盐为催化剂, 在 $300{ }^{\circ} \mathrm{C}, 25 \mathrm{MPa}$ 下催化果糖反应可得 $40 \%$ ～50\%的乳 酸 ${ }^{[23,24]}$, 该方法对生产设备要求较高, 催化剂不容易回 收利用, 产物分离困难. 在 $190{ }^{\circ} \mathrm{C}, 5 \mathrm{MPa}$ 的 $\mathrm{He}$ 压力下, 以沸石, $\mathrm{C}-\mathrm{SO}_{3} \mathrm{H}, \mathrm{ZrOS}, \mathrm{AlW}, \mathrm{ZrW}$, 杂多酸等非均相催 化剂, 催化纤维素制备乳酸, 最高收率为 $28 \%{ }^{[25,26]}$, 该 体系反应条件仍比较苛刻, 乳酸收率在 $30 \%$ 以下. 为此, 寻找节能、环保、高效的乳酸制备方法引起了人们的关 注.

离子液体的催化活性较高, 其反应产物中没有难以 除去的阳离子, 可以替代无机盐催化剂 ${ }^{[27,28]}$; 溶解能力 强、热稳定性高、难以氧化、毒性较低、蒸汽压低、对 环境友好、易回收可循环使用、可以设计, 有 “绿色溶

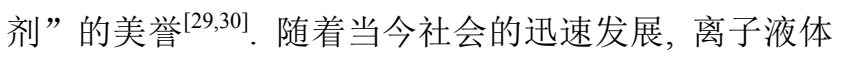
相关的研究吸引了学者的眼球 ${ }^{[31,32]}$. 将离子液体单体聚 合成为高分子聚合物, 其高分子链上至少含有一个离子 中心, 形成具有更加优越性能的功能高分子一一聚合离 子液体 ${ }^{[33]}$. 聚合离子液体作为一种综合了高分子与离 子液体性质的功能高分子材料 ${ }^{[31,34,35]}$, 在离子导体、吸 附与分离、分散剂以及催化剂等方面具有广阔的应用前
景 ${ }^{[33]}$. 目前, 对聚合离子液体的研究主要集中在对 $\mathrm{CO}_{2}$ 的吸附方面 ${ }^{[36]}$, 而其在糖类催化转化制备乳酸方面却 鲜有报道.

本文对以 $[\mathrm{IMEP}] \mathrm{Cl}$ 作为催化剂催化转化果糖制备 乳酸的反应条件进行了研究，探讨了聚合离子液体和碱 类型、反应温度、 $[\mathrm{IMEP}] \mathrm{Cl}$ 和碱用量、底物浓度以及气 体氛围等条件对乳酸收率的影响, 优化了反应的条件, 并对该体系催化转化果糖制备乳酸的反应路径进行了 初步的推测.

\section{1 结果与讨论}

\section{1 催化剂和固体碱的优化选择}

分别选用 $[\mathrm{BMIM}] \mathrm{Cl},[\mathrm{OMIM}] \mathrm{Cl},[\mathrm{EMIM}] \mathrm{Cl}$ 离子液 体及 $[\mathrm{IMEP}] \mathrm{Cl},[\mathrm{IMEP}] \mathrm{BF}_{4},[\mathrm{IMEP}] \mathrm{ClO}_{4},[\mathrm{IMEP}] \mathrm{PhCOO}$, [IMEP]Ac 等含有不同阴离子的聚合离子液体作为催化 剂. $\mathrm{NaOH}, \mathrm{KOH}, \mathrm{Ca}(\mathrm{OH})_{2}$ 及 $\mathrm{Ba}(\mathrm{OH})_{2}$ 提供碱性环境, 催 化转化果糖制备乳酸.

由表 1 可知, 不同的离子液体、聚合离子液体和固 体碱对乳酸的收率有较大影响.

表 1 不同(聚合)离子液体及碱对乳酸收率的影响 ${ }^{a}$

Table 1 Yield of lactic acid with different (polymer) ionic liquid in various alkaline solutions

\begin{tabular}{|c|c|c|c|c|c|}
\hline 序号 & 碱 & 催化剂 & $\begin{array}{c}\text { 碱浓度/ } \\
\left(\mathrm{mmol} \cdot \mathrm{L}^{-1}\right)\end{array}$ & $\begin{array}{c}\text { 果糖 } \\
\text { 转化率/\% }\end{array}$ & $\begin{array}{c}\text { 乳酸 } \\
\text { 收率 } / \%\end{array}$ \\
\hline 1 & $\mathrm{NaOH}$ & - & 50 & 89.2 & 23.3 \\
\hline 2 & $\mathrm{NaOH}$ & [BMIM]Cl & 50 & 83.5 & 11.3 \\
\hline 3 & $\mathrm{NaOH}$ & [OMIM]Cl & 50 & 92.4 & 36.1 \\
\hline 4 & $\mathrm{NaOH}$ & [EMIM]Cl & 50 & 85.2 & 11.7 \\
\hline 5 & $\mathrm{NaOH}$ & [IMEP]Cl & 50 & 98.8 & 65.0 \\
\hline 6 & $\mathrm{NaOH}$ & {$[\mathrm{IMEP}] \mathrm{BF}_{4}$} & 50 & 98.0 & 61.2 \\
\hline 7 & $\mathrm{NaOH}$ & {$[\mathrm{IMEP}] \mathrm{ClO}_{4}$} & 50 & 98.6 & 55.4 \\
\hline 8 & $\mathrm{NaOH}$ & [IMEP]phCOO & 50 & 98.4 & 35.7 \\
\hline 9 & $\mathrm{NaOH}$ & [IMEP]Ac & 50 & 96.5 & 45.8 \\
\hline 10 & $\mathrm{KOH}$ & [IMEP]Cl & 50 & 99.1 & 67.3 \\
\hline 11 & $\mathrm{Ca}(\mathrm{OH})_{2}$ & [IMEP]Cl & 25 & 97.3 & 37.1 \\
\hline 12 & $\mathrm{Ba}(\mathrm{OH})_{2}$ & [IMEP]Cl & 25 & 97.2 & 40.6 \\
\hline
\end{tabular}

${ }^{a}$ Reaction conditions: a mixture of fructose $(25 \mathrm{mmol} / \mathrm{L})$, ploymer ionic liquid $(100 \mathrm{mmol} / \mathrm{L})$, water $(40 \mathrm{~mL}), \mathrm{N}_{2}(2 \mathrm{MPa}), 100{ }^{\circ} \mathrm{C}$.

传统离子液体是离子液体单体, 但聚合离子液体是 将离子液体单体聚合成为高分子聚合物, 其高分子链上 至少含有一个离子中心, 因此具有更优越的功能. 在该 体系中(表 1,2 9 行), 聚合离子液体的催化效果明显优 于离子液体的催化效果, 其可能原因是聚合离子液体链 在水中充分展开, 每条聚合离子液体链上的多个活性中 心 $\mathrm{Imi}^{+}$均对本体系中催化果糖制备乳酸有促进作用.

[IMEP]Cl 的催化效果明显优于含有其它阴离子的 聚合离子液体(表 1,5 9 行), 在其催化作用下乳酸的收 率最高为 $65 \%,[\mathrm{IMEP}] \mathrm{BF}_{4}$ 的催化效果仅次于 $[\mathrm{IMEP}] \mathrm{Cl}$ 
为 $61.2 \%,[\mathrm{IMEP}] \mathrm{ClO}_{4},[\mathrm{IMEP}] \mathrm{Ac}$ 次之, [IMEP]PhCOO 的催化效果最差为 $35.7 \%$. 一个可能的原因是 $[\mathrm{IMEP}]^{+}$ 阳离子中的 $2 \mathrm{C}-\mathrm{H}$ 与 $\mathrm{Cl}^{-}$在本体系中形成了氢键, 强的氢 键作用使得咪唑中的 $2 \mathrm{C}-\mathrm{H}$ 易于活化, 从而显著增强了 $2 \mathrm{C}-\mathrm{H}$ 的酸性. 本体系通过多步酸碱催化脱水反应和水 合反应催化果糖制备乳酸, $\mathrm{OH}^{-}$的加入更有助于协同 $[\mathrm{IMEP}] \mathrm{Cl}$ 催化果糖制备乳酸, 故 [IMEP] $\mathrm{Cl}$ 的催化效果 最好 ${ }^{[37 \sim 39]}$. 另一个可能的原因是 5 种阴离子与 [IMEP] ${ }^{+}$ 在水中的结合能力不同, 从弱到强依次是 $\mathrm{Cl}^{-}<\mathrm{BF}_{4}^{-}<$ $\mathrm{ClO}_{4}^{-}<\mathrm{Ac}^{-}<\mathrm{PhCOO}^{-}$. 在该反应体系中, 当 $\mathrm{Cl}^{-}$存在 时, [IMEP $]^{+}$在水中能充分展开, 从而使得体系中的正电 荷的密度升高, 进而其链周围的 $\mathrm{OH}^{-}$离子及反应物浓 度升高 ${ }^{[40]}$, 故 [IMEP $] \mathrm{Cl}$ 的催化效果最好. 以 $[\mathrm{IMEP}] \mathrm{Cl}$ 为催化剂, 在 $\mathrm{KOH}$ 溶液中催化果糖制备乳酸最有利, 乳酸的收率在 $30 \mathrm{~min}$ 内达到 $67.3 \%$; $\mathrm{NaOH}$ 提供碱性环 境的反应体系中乳酸的收率次之, 收率最高为 $65 \%$. $\mathrm{Ca}(\mathrm{OH})_{2}$ 与 $\mathrm{Ba}(\mathrm{OH})_{2}$ 体系中乳酸的收率较低的原因是 $\mathrm{Ca}(\mathrm{OH})_{2}$ 与 $\mathrm{Ba}(\mathrm{OH})_{2}$ 的溶解度相对 $\mathrm{NaOH}, \mathrm{KOH}$ 较低, 溶 液碱性较弱. 虽然 $\mathrm{KOH}$ 在本体系中对乳酸的生成最有 利, 但是 $\mathrm{KOH}$ 的价格相对 $\mathrm{NaOH}$ 高, 在生产中 $\mathrm{NaOH}$ 的使用更为广泛, 所以综合考虑各因素, 选择 $\mathrm{NaOH}$ 用 以探索 $[\mathrm{IMEP}] \mathrm{Cl}$ 的催化能力.

因此，本文选用[IMEP]Cl 为催化剂，在 $\mathrm{NaOH}$ 溶液 体系中催化转化果糖制备乳酸.

\section{2 不同温度对乳酸收率的影响}

由图 1 可知, 随着反应温度的升高, 乳酸的收率均 呈现出先升高再降低的趋势. 在 $100{ }^{\circ} \mathrm{C}$ 时乳酸达到最高 收率 $65 \%$. 反应温度过低, 不利于果糖中链的裂解和乳 酸的生成; 而反应温度过高, 副反应较多, 也使得乳酸 收率降低.

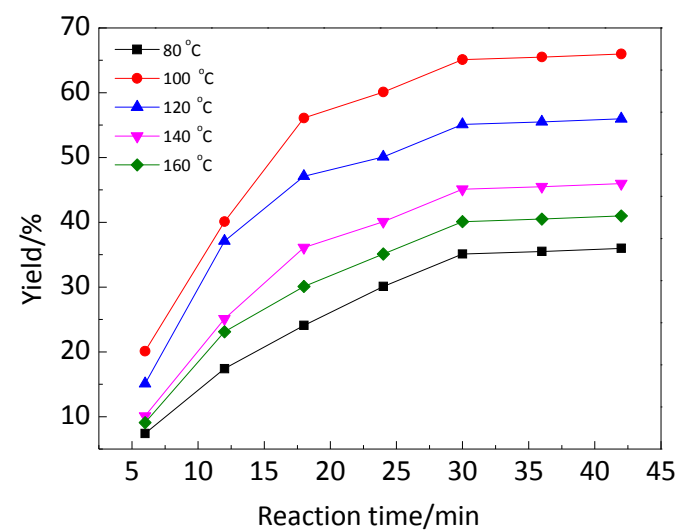

图 1 不同温度下乳酸收率随时间的变化

Figure 1 Yield of lactic acid with different temperatures Reaction conditions: a mixture of fructose $(25 \mathrm{mmol} / \mathrm{L})$, [IMEP]Cl (100 $\mathrm{mmol} / \mathrm{L}), \mathrm{NaOH}(50 \mathrm{mmol} / \mathrm{L})$, water $(40 \mathrm{~mL}), \mathrm{N}_{2}(2 \mathrm{MPa})$

\section{3 [IMEP]Cl, $\mathrm{NaOH}$ 浓度对乳酸收率的影响}

在 $100{ }^{\circ} \mathrm{C}$ 下，考察了 $[\mathrm{IMEP}] \mathrm{Cl}$ 和 $\mathrm{NaOH}$ 浓度对乳酸 收率的影响. 由图 2 可知, 在该体系中, 随着 $\mathrm{NaOH}$ 的 增加, 乳酸的收率先增加后减少, 当 $\mathrm{NaOH}$ 的浓度为 50 $\mathrm{mmol} / \mathrm{L}$ 时, 乳酸的最高收率达到 $65 \%$. 其原因是 $\mathrm{OH}^{-}$ 浓度过低时，体系中的碱性较弱，不利于该体系中乳酸 的生成，而过量的 $\mathrm{OH}^{-}$又阻碍了 [IMEP]Cl 对本体系的 催化作用.

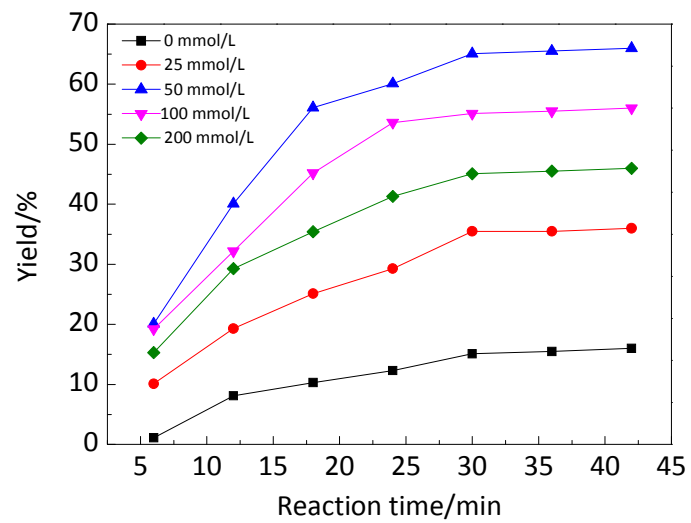

图 $2 \mathrm{NaOH}$ 浓度对乳酸收率的影响

Figure 2 Yield of lactic acid with different concentration of $\mathrm{NaOH}$

Reaction conditions: a mixture of fructose $(25 \mathrm{mmol} / \mathrm{L}),[\mathrm{IMEP}] \mathrm{Cl}(100$ $\mathrm{mmol} / \mathrm{L})$, water $(40 \mathrm{~mL}), \mathrm{N}_{2}(2 \mathrm{MPa}), 100{ }^{\circ} \mathrm{C}$

由图 3 可知, 随着 $[\mathrm{IMEP}] \mathrm{Cl}$ 的浓度的增大, 果糖的 转化率逐渐升高, 而乳酸的收率呈现出先升高再降低的 趋势. 当 $[\mathrm{IMEP}] \mathrm{Cl}$ 的浓度为 $100 \mathrm{mmol} / \mathrm{L}$ 时, 乳酸的收 率最高, 可能原因是当 [IMEP]Cl 的浓度过低时, 其催化 活性较低, 不利于乳酸的生成; 当[IMEP]Cl 浓度过高 时，体系中会有大量的副反应，生成大量的黄褐色腐殖

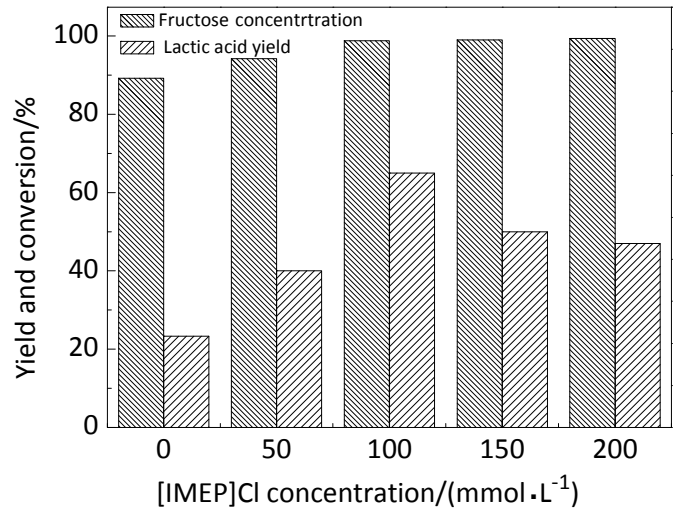

图 $3[\mathrm{IMEP}] \mathrm{Cl}$ 浓度对乳酸果糖转化率、乳酸收率的影响

Figure 3 Concentration of fructose and yield of lactic acid with different concentration of [IMEP]Cl

Reaction conditions: a mixture of fructose $(25 \mathrm{mmol} / \mathrm{L}), \mathrm{NaOH}(50$ $\mathrm{mmol} / \mathrm{L})$, water $(40 \mathrm{~mL}), \mathrm{N}_{2}(2 \mathrm{MPa}), 100{ }^{\circ} \mathrm{C}$ 
物, 从而导致乳酸的收率降低.

综合以上结果可知, 当体系中 $n_{\mathrm{NaOH}}: n_{[\mathrm{IMEP}] \mathrm{Cl}}=1$ : $2(\mathrm{NaOH} 50 \mathrm{mmol} / \mathrm{L}$, [IMEP]Cl $100 \mathrm{mmol} / \mathrm{L})$ 时, 最有利 于乳酸的生成, 其最高收率为 $65 \%$.

\section{4 不同底物浓度对乳酸收率的影响}

由图 4 可知, 随着底物浓度的增加, 乳酸的收率逐 渐降低. 在底物浓度较低时, 底物能与 $[\mathrm{IMEP}] \mathrm{Cl}$ 充分接 触，从而使得反应完全; 当反应体系中底物浓度升高, 体系中催化剂和底物的相对浓度降低, 从而使得在单位 体积内催化剂的催化活性降低, 不能充分催化反应物, 必然导致乳酸收率的降低.

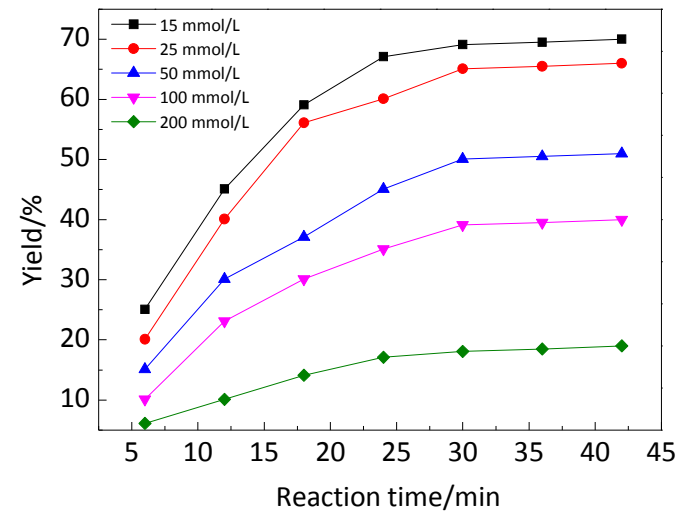

图 4 不同底物浓度下乳酸的收率随时间的变化

Figure 4 Yield of lactic acid with different concentrations of fructose

Reaction conditions: a mixture of [IMEP]Cl $(100 \mathrm{mmol} / \mathrm{L}), \mathrm{NaOH}(50$ $\mathrm{mmol} / \mathrm{L})$, water $(40 \mathrm{~mL}), \mathrm{N}_{2}(2 \mathrm{MPa}), 100{ }^{\circ} \mathrm{C}$

\section{5 不同的气体氛围对乳酸收率的影响}

在反应体系中分别通入 $2 \mathrm{MPa}$ 的空气, $\mathrm{N}_{2}, \mathrm{O}_{2}$, 考察 在不同的气体氛围中乳酸的收率随时间的变化. 由图 5 可知, 不同的气体氛围对乳酸的生成有较大的影响. $\mathrm{N}_{2}$ 氛围下有利于乳酸生成, 空气次之, $\mathrm{O}_{2}$ 氛围最不利. 因 为在有氧条件下, 反应过程中的一部分中间产物被氧 化, 从而使副反应增多, 不利于乳酸生成; 一部分产物 也会被氧化生成其他物质, 从而使得乳酸的收率降低. 故本文选择在 $\mathrm{N}_{2}$ 氛围下催化果糖制备乳酸.

\section{6 果糖转化为乳酸的机理}

该反应体系催化果糖转化制备乳酸的效果明显优 于只用 $\mathrm{NaOH}$ 的体系, 其催化效果较理想的原因是由于 在水中 $[\mathrm{IMEP}] \mathrm{Cl}$ 的链能够充分展开, 并能以 $\mathrm{Imi}^{+}{ }^{+}$链为 中心通过静电作用力吸附游离的 $\mathrm{OH}^{-}$, 使其周围的 $\mathrm{OH}^{-}$浓度明显高于体系中 $\mathrm{OH}^{-}$浓度, 从而使得果糖在一 个 $\mathrm{OH}^{-}$浓度相对较高的局部环境下转化为乳酸(图 5).

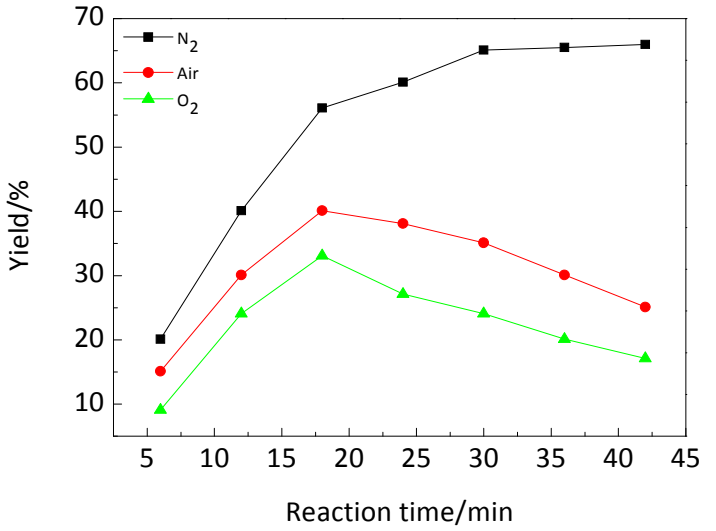

图 5 不同气体氛围下乳酸收率随时间的变化曲线

Figure 5 Yield of lactic acid under different gases atmosphere Reaction conditions: a mixture of fructose $(25 \mathrm{mmol} / \mathrm{L})$, [IMEP]Cl (100 $\mathrm{mmol} / \mathrm{L}), \mathrm{NaOH}(50 \mathrm{mmol} / \mathrm{L})$, water $(40 \mathrm{~mL}), \mathrm{N}_{2}(2 \mathrm{MPa}), 100{ }^{\circ} \mathrm{C}$

在 $100{ }^{\circ} \mathrm{C}$ 下，利用 HPLC 对反应过程中的糖浓度 进行监测, 可以得到图 6 的结果, 反应过程中随着果糖 浓度的减少, 有少量葡萄糖生成, 且生成量呈现出先增 加后减少的趋势, 由此表明, 果糖转化为乳酸的过程中, 会部分地转化为葡萄糖.

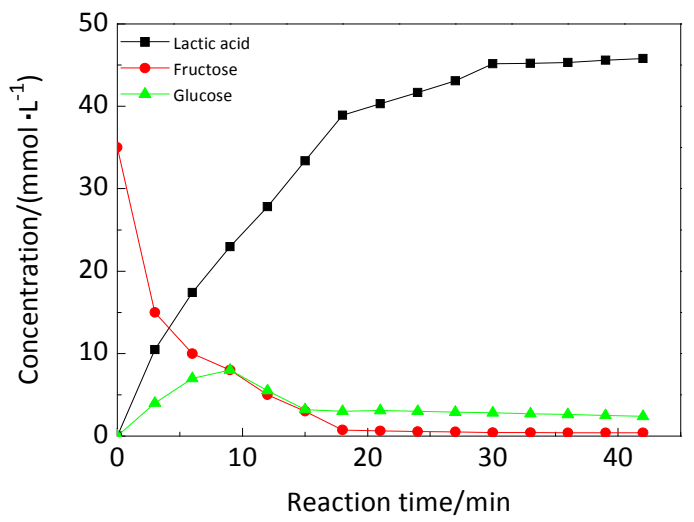

图 6 反应体系中果糖、葡萄糖和乳酸浓度随时间的变化趋势 Figure 6 The change of fructose, glucose and lactic acid concentration with the time

Reaction conditions: a mixture of fructose $(25 \mathrm{mmol} / \mathrm{L})$, [IMEP]Cl (100 $\mathrm{mmol} / \mathrm{L}), \mathrm{NaOH}(50 \mathrm{mmol} / \mathrm{L})$, water $(40 \mathrm{~mL}), \mathrm{N}_{2}(2 \mathrm{MPa}), 100{ }^{\circ} \mathrm{C}$

该体系中果糖催化转化制备乳酸可能经历了如下 途径: 果糖和葡糖糖之间可以相互转化, 生成乳酸的过 程中果糖首先水解生成甘油醛和二羟基丙酮, 然后二羟 基丙酮和甘油醛经过脱水与烯-醇异构生成丙酮醛，最 后丙酩醛经加水和 1,2 -氢转移生成乳酸 ${ }^{[1,42]}$ (Scheme 2).

该体系中影响果糖制备乳酸的收率的关键步骤是 二差基丙酮生成丙酮醛的过程 ${ }^{[43]}$. 推测 [IMEP]Cl 对本 体系的催化作用主要表现在由二羟基丙酮到丙酮醛的 过程. 其可能的催化原因是咪唑环上的氢在该体系中相 当于 1 个 $L$-酸中心, 能够吸引其周围的 $\mathrm{OH}^{-}$, 使得二羟 


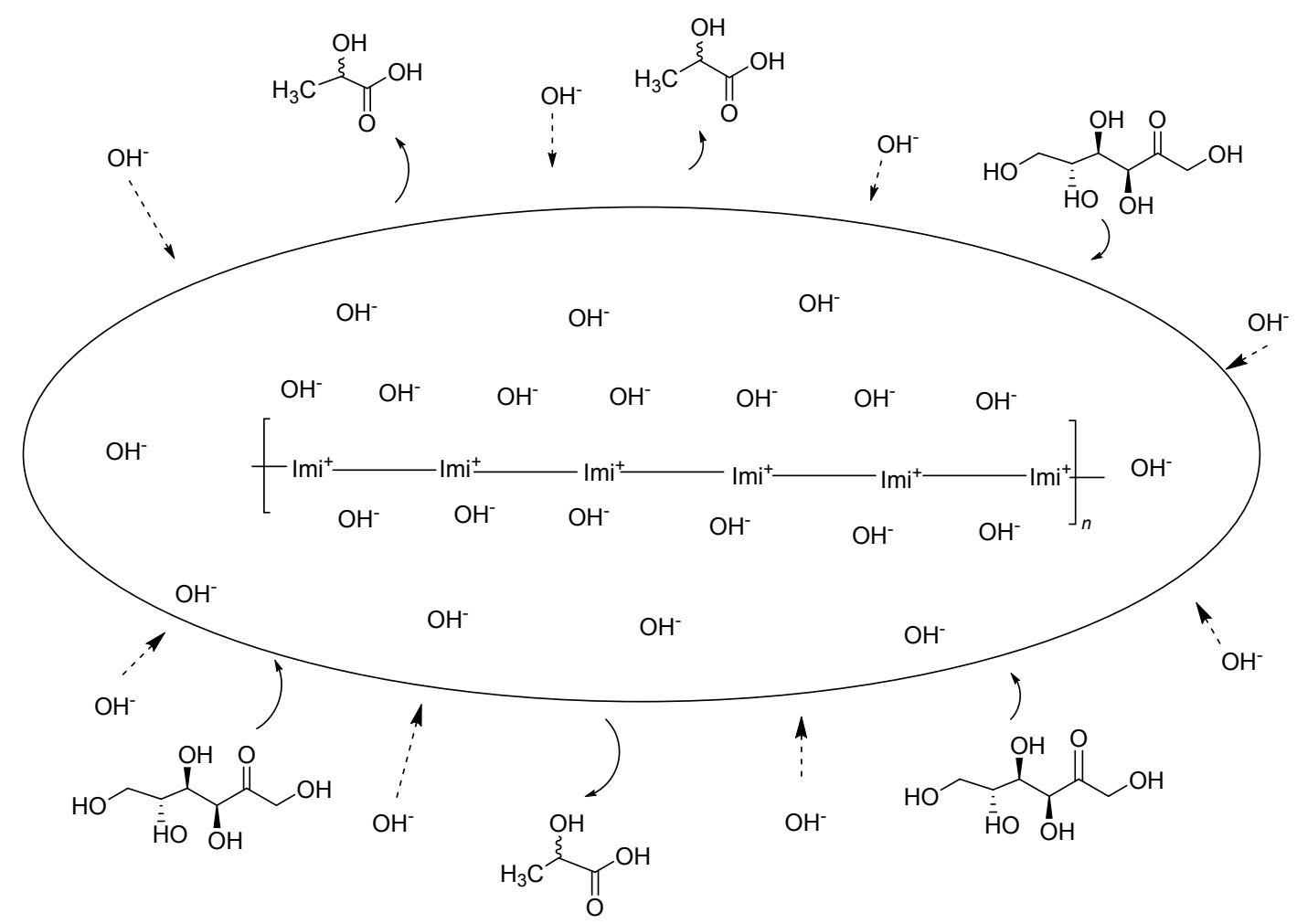

图式 1 [IMEP]Cl 催化作用示意图

Scheme 1 The schematic diagram of [IMEP]Cl<smiles>C=CC=CC(O)C(O)[C@H](O)[C@H](O)[C@H](O)CO</smiles><smiles>O=CC(O)CO</smiles>
$D$-glyceraldehyde D-dihydroxyacetone<smiles>CC(=O)C=CC(=O)O[C@H](C)C(=O)O</smiles>

图式 2 果糖转化为乳酸的反应路径

Scheme 2 Conversion of $D$-glucose into lactic acid

基丙酮在局部较高的碱浓度下经脱水和烯一醇异构生成 甘油醛.

果糖在碱性水热条件下制备催化转化制备乳酸, 会 有较多的副产物的生成. 用 GC-MS 对产物进行检测, 副产物较多的组分有乙醇酸、2-羟基丁酸、3-差基丙酸, 2-羟基-2-丁烯酸、甘油、2,3-二羟基丙酸、2,4-二羟基丁 酸、3,4-二羟基丁酸, 4-着基丁酸、苏糖酸等.

\section{$1.7[\mathrm{IMEP}] \mathrm{Cl}$ 的循环利用}

[IMEP]Cl 在催化分解果糖制备乳酸的过程中表现

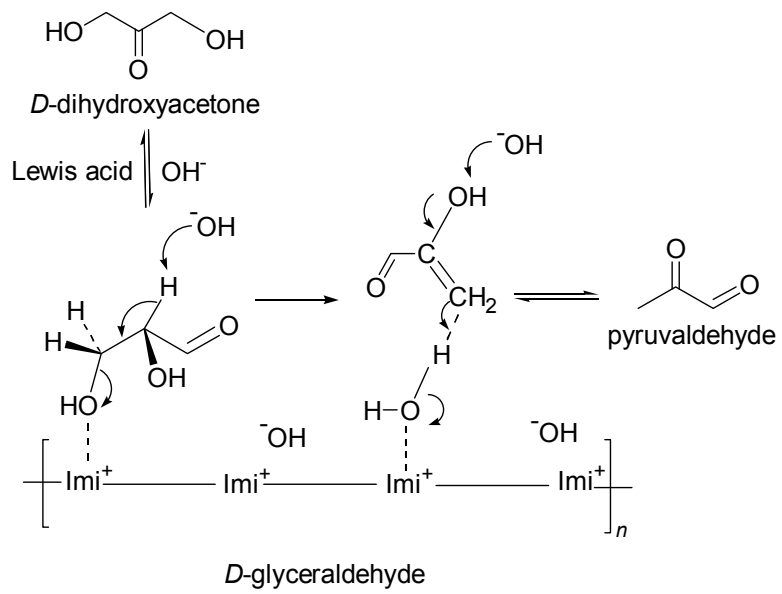

图式 3 [IMEP]Cl 的催化机理

Scheme 3 The catalytic mechanism of [IMEP]Cl

出了良好的催化活性, 能显著降低反应的温度和压力, 研究其重复利用性能对后期可能的工业应用具有比较 积极的指导意义. 在该实验中，对反应产物用等体积的 正丁醇萃取并离心, 取下层萃余液, 加入一定量的果 糖、 $\mathrm{NaOH}$ 继续进行反应，在相同条件下循环使用 5 次， 得到的乳酸收率仍在 $55 \%$ 以上(图 7). 以上结果表明 $[\mathrm{IMEP}] \mathrm{Cl}$ 具有很好的循环利用性能. 


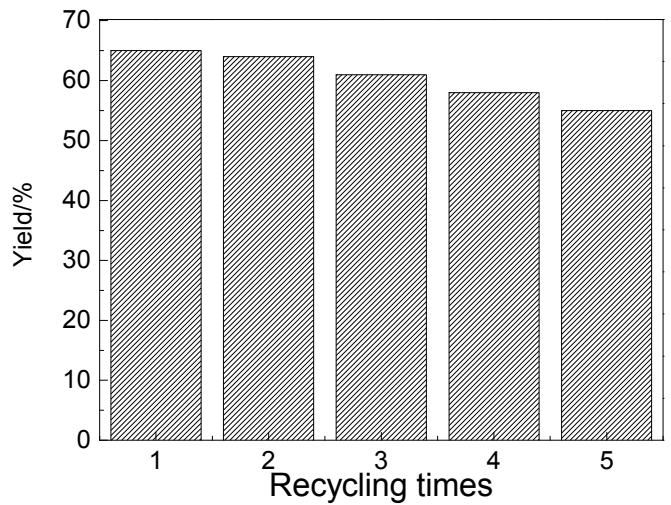

图 7 [IMEP]Cl 的循环利用

Figure 7 Recycling of the ploymer ionic liquid

\section{2 结论}

在 $\mathrm{NaOH}$ 碱溶液中, [IMEP]Cl 能够非常有效地催化 分解果糖制备乳酸. 在 $100{ }^{\circ} \mathrm{C}$ 条件下, 果糖 $25 \mathrm{mmol} / \mathrm{L}$, [IMEP]Cl $100 \mathrm{mmol} / \mathrm{L}, \mathrm{NaOH} 50 \mathrm{mmol} / \mathrm{L}$, 去离子水 40 $\mathrm{mL}$, 反应 $30 \mathrm{~min}$ 后乳酸达到最高收率 $65 \%$. 通过对气 体氛围的研究发现, 无氧条件更有利用乳酸的生成. $[\mathrm{IMEP}] \mathrm{Cl}$ 经过 5 次回收利用, 仍然可以达到较好的效 果. 对果糖生成乳酸的反应机理进行了初步的推测. 该 研究为今后进一步探究碳水化合物分解制备乳酸有较 好的借鉴意义.

\section{3 实验部分}

\section{1 仪器与试剂}

美国瓦里安公司 ProStar 高效液相色谱仪; 德国 Bruck 公司 TENSONR207 傅里叶变换红外光谱仪; Bruck AV600 超导液体核磁共振谱仪; 日本岛津公司 $\mathrm{GC} / \mathrm{MS}$ 气相色谱质谱联用仪; 上海精密科学仪器有限 公司 ZD-2 型全自动电位滴定仪.

咪唑(AR), 环氧氯丙烷(AR), 果糖(AR), 氢氧化钠 $(A R)$, 氢氧化钾 $(A R)$, 氢氧化钙 $(A R)$, 氢氧化钡 $(A R)$, 嗍氟酸钠 (AR), 一水高氯酸锂 (AR), 乙酸钠 $(A R)$, 苯甲 酸钠 $(A R)$, 乳酸 $(A R)$, 葡萄糖 $(A R)$, 磷酸二氢钠 $(A R)$, 乙腈(AR), 去离子水.

\section{2 实验方法}

采用高效液相色谱(HPLC), 对乳酸、果糖含量进行 检测. 乳酸的色谱检测条件: 紫外吸收检测器, $\mathrm{C}_{18}$ 色谱 柱, 流动相 $[$ 磷酸缓冲液 $(\mathrm{pH}=2):$ 乙腈 $=95: 5$, 体积 比], 流速 $1 \mathrm{~mL} / \mathrm{min}$, 检测波长 $210 \mathrm{~nm}$; 果糖的色谱检 测条件: 折光示差检测器, $\mathrm{NH}_{2}$ 色谱柱, 流动相(乙腈 : 水 $=77: 23$, 体积比), 流速 $1 \mathrm{~mL} / \mathrm{min}$. 用峰面积归一法 计算乳酸收率、果糖转化率.
[IMEP]Cl 的制备参见文献[28]. 准确称取 $1 \mathrm{~mol}$ 的 咪唑于 $1 \mathrm{~L}$ 的三口烧瓶中, $1 \mathrm{~mol}$ 的环氧氯丙烷于恒压漏 斗中, 加入适量的乙醇作溶剂, 在 $30{ }^{\circ} \mathrm{C}$ 的恒温条件下 搅拌并缓慢滴加环氧氯丙烷于三口烧瓶中, 待滴加完毕 后继续反应 $2 \mathrm{~h}$, 将反应温度上升至 $45{ }^{\circ} \mathrm{C}$ 继续反应 12 $\mathrm{h}$, 最后把温度升至 $85{ }^{\circ} \mathrm{C}$ 反应 $72 \mathrm{~h}$, 反应结束后将反应 产物冷却, 抽滤, 并用无水乙醇洗涤至少 5 次, 真空干 燥 $12 \mathrm{~h}$, 备用.

不同阴离子的聚合离子液体的制备：准确称量 0.25 $\mathrm{mol}$ [IMEP]Cl, $0.25 \mathrm{~mol}$ 喼氟酸钠(高氯酸锂、醋酸钠、 苯甲酸钠)于 $1 \mathrm{~L}$ 三口烧瓶中, 加入 $300 \mathrm{~mL}$ 无水乙醇, 将三口烧瓶置于 $85{ }^{\circ} \mathrm{C}$ 恒温油浴中搅拌反应 $24 \mathrm{~h}$, 反应 结束后将产物冷却, 抽滤, 并用无水乙醇洗涤至少 5 次, 真空干燥 $12 \mathrm{~h}$, 备用.

[IMEP]Cl 的表征: 通过红外、核磁对 [IMEP]Cl 进行 了表征 ${ }^{[28]}$. ${ }^{1} \mathrm{H}$ NMR $\left(\mathrm{D}_{2} \mathrm{O}, 600 \mathrm{MHz}\right) \delta: 4.3(2 \mathrm{H}), 4.4,4.6$ $(2 \mathrm{H}), 7.8(2 \mathrm{H}) ;{ }^{13} \mathrm{C}$ NMR $\left(\mathrm{D}_{2} \mathrm{O}, 150 \mathrm{MHz}\right) \delta: 54,69,125$, 148; IR (KBr) v: 3485, 3167, 3126, 2969, 2877, 1579, $1470 \mathrm{~cm}^{-1}$

果糖催化转化制备乳酸: 称量 $0.18 \mathrm{~g}$ 果糖, $0.08 \mathrm{~g}$ 氢 氧化钠, $0.64 \mathrm{~g}$ [IMEP] Cl, $40 \mathrm{~mL}$ 去离子水于耐高压反应 釜中, 在 $100{ }^{\circ} \mathrm{C}, 2 \mathrm{MPa}$ 氮气氛围中反应. 反应结束后 将反应产物调节为酸性并过滤，用高效液相色谱分别检 测乳酸、果糖的含量.

收率计算:

$y($ 乳酸收率 $)=n($ 目标产物中碳原子的物质的量 $) /$

$n$ (反应物中碳原子的物质的量) $\times 100 \%$

转化率计算:

$x($ 果糖转化率 $)=[n($ 反应前果糖的物质的量 $)-n($ 反应 后果糖的物质的量 $)] / n$ (反应前果糖的物质的量 $) \times$ $100 \%$

辅助材料(Supporting Information) 所合成的聚合离 子液体的红外谱图, 反应体系的 CC-MS 谱图. 这些材 料可以免费从本刊网站(http://sioc-journal.cn/)上下载.

\section{References}

[1] Keshwani, D. R.; Cheng, J. J. Bioresour. Technol. 2009, 100(4), 1515 .

[2] Sims, R. E. H.; Mabee, W.; Saddler, J. N.; Taylor, M. Bioresour. Technol. 2010, 101(6), 1570.

[3] Rom, L. Y.; Barrettc, J.; Liu, Z. Y. Nat. Biotechnol. 2007, 447, 982.

[4] Chang, C. Ph.D. Dissertation, Institute of Bioengineering, Zhejiang University, Hangzhou, 2006 (in Chinese). (常春, 博士论文, 浙江大学生物工程研究所, 杭州, 2006.)

[5] Zhu, G.-Y.; Ma, Y.-H.; Zhu, X. Chin. J. Org. Chem. 2010, 30, 142 
(in Chinese).

(朱广用, 马艳华, 朱宪, 有机化学, 2010, 30, 142.)

[6] Huber, G. W.; Iborra, S.; Corma, A. Chem. Rev. 2006, 106, 4044.

[7] Demirbas, M. F.; Balat, M. Energy Convers. Manage. 2006, $47(15 \sim 16), 2371$.

[8] Gallezot, P. Chem. Soc. Rev. 2012, 41, 1538.

[9] Chandel, A. K.; da Silva, S. S.; Carvalho, W.; Singh, O. V. J. Chem. Technol. Biotechnol. 2012, 87(1), 11.

[10] Liu, Z. Ph.D. Dissertation, Zhejiang University, Hangzhou, 2012 (in Chinese). (刘镇, 博士论文, 浙江大学, 杭州, 2012.)

[11] San-Mart in, M.; Pazos, C.; Coca, J. J. Chem. Technol. Biotechnol. 1992, 54, 1e6.

[12] Epane, G.; Laguerre, J. C.; Wadouachi, A.; Marek, D. Green Chem. 2010, 12(3), 502.

[13] Gao, C.; Ma, C.; Xu, P. Biotechnol. Adv. 2011, 29(6), 930.

[14] Bai, D.; Zhao, X.; Li, X.; Xu, S. Biochem. Eng. J. 2004, 18(1), 41.

[15] Salminen, S.; Ouwehand, A.; Wright, A. V.; Daly, C. Ann. N. Y. Acad. Sci. 1993, 429.

[16] Zeng, W.; Chen, F.-Q.; Zhan, X.-L; Chem. Ind. Eng. Progr. 2006, 25(7), 744 (in Chinese).

(曾炜, 陈丰秋, 詹晓力, 化工进展 2006, 25(7), 744.)

[17] Wasewar, K. L.; Yawalkar, A. A.; Moulijn, J. A.; Pangarkar, V. G. Ind. Eng., Chem. Res. 2004, 43(19), 5969.

[18] Aida, T. M.; Tajima, K.; Watanabe, M.; Saito, Y.; Kuroda, K.; Nonaka, T.; Hattori, H.; Smith, R. L.; Arai, K. J. Supercrit. Fluids 2007, 42(1s), 110 .

[19] Onda, A.; Ochi, T.; Kajiyoshi, K.; Yanagisawa, K. Catal. Commol. 2008, 9(6), 1050.

[20] Onda, A.; Ochi, T.; Kajiyoshi, K.; Yanagisawa, K. Appl. Catal. A 2008, 343(1 2), 49.

[21] Yan, X.-Y.; Jin, F.-M.; Tohji, K.; Kishita, A.; Enomoto, H. AIChE J. 2010, 56, 2727.

[22] Zhang, S.-P.; Jin, F. -M.; Hu, J.-J.; Huo, Z.-B. Bioresour. Technol. 2010, 102(2011), 1998.

[23] Rasrendra, C. B.; Makertihartha, I.; Adisasmito, S.; Heeres, H. J. Top. Catal. 2010, 53(15 18), 1241.

[24] Hayashi, Y.; Sasaki, Y. Chem. Commol/Lun. 2005, 41(21), 2716 。

[25] Holm, M. S.; Saravanamurugan, S.; Taarning, E. Science 2010, 328,602 .

[26] Chambon, F.; Rataboul, F.; Pinel, C.; Cabiac, A.; Guillon, E.; Essayem, N. Appl. Catal. B 2011, 105, 171.
[27] (a) Aslanov, L. A.; Zakharov, V. N.; Savilov, S. V. RU 2471709, 2013 [Chenm. Abstr. 2013, 158, 162458].

(b) Patil, A. B.; Pathak, P.; Shinde, V. S.; Godbole, S. V.; Mohahapatra, P. K. Dalton Trans. 2013, 42(5), 1519.

(c) Luo, S.-Z.; Mi, X.-L.; Zhang, L.; Liu, S.; Xu, H.; Cheng, J.-P. Tetrahedron 2007, 1923.

[28] Song, Y.-L.; Wang, X.-C.; Huang, C.-P.; Liang, F.-B.; Chen, B.-H. Chin. J. Org. Chem. 2013, 33, 1715 (in Chinese).

(宋彦磊, 王新承, 黄崇品, 梁凤兵, 毓志超, 陈标华, 有机化学, 2013, 33, 1715.)

[29] Gao, Y.-J. M.S. Thesis, Zhejiang Normal University, Jinhua, 2009 (in Chinese).

(高尤剑，硕士论文，浙江师范大学，金华, 2009.)

[30] Wei, Z.-Z. M.S. Thesis, Shandong University, Jinan, 2012 (in Chinese). (韦珍珍, 硕士论文, 山东大学, 济南, 2012.)

[31] Li, J.; Sun, H.; Cai, X.-C.; Dai, L.-Y. Chin. J. Org. Chem. 2007, 27, 1296 (in Chinese).

(李娟, 孙辉, 蔡晓晨, 戴立益, 有机化学, 2007, 27, 1296.)

[32] Hirao, M.; Ito, K.; Ohno, H. Electrochim. Acta 2000, 45, 1291.

[33] Shaplov, A. S.; Lozinskaya, E. I.; Ponkratov, D. O.; Malyshkina, I. A.; Vidal, F.; Aubert, P. H.; Okatova, O. V.; Pavlov, G. M.; Komarova, L. I.; Wandrey, C.; Vygodskii, Y. S. Electrochim. Acta 2011, 57,74 .

[34] Ohno, H.; Ito, K. Chem. Lett. 1998, 27, 751.

[35] Hirao, M.; Ito, K.; Ohno, H. Polym. Adv. Technol. 2000, 11, 534.

[36] Zhang, H.; Zhou, Z. -B.; Nie, J. Prog. Chem. 2013, 25(5), 761 (in Chinese).

(张恒, 周志涁, 聂进, 化学进展, 2013, 25(5), 761.)

[37] He, H.-Y.; Zhang, S.-J.; Liu, X.-M.; Wang, J.-W.; Yao, X.-Q.; Zhang, X.-P. Fluid Phase Equilib. 2013, 169.

[38] Yan, R.-Y.; Yang, P.; Li, Z.-X.; Wang, H.; Diao, Y.-Y.; Wang, L.; Zhang, S.-J. Adv. Mater. Res. 2011, 1146.

[39] Dong, K.; Song, Y.-T.; Liu, X.-M.; Cheng, W.-G.; Yao, X. -Q.; Zhang, S.-J. J. Phys. Chem. B 2011, 1007.

[40] Kanazawa, A.; Ikeda, T.; Endo, T. J. Polym. Sci., Part A: Polym. Chem. 1993, 31, 1099.

[41] Bicker, M.; Endres, S.; Ott, L.; Vogel, H. Mol. J. Catal. A: Chem. 2005, 239, 151.

[42] Ellis, A. V.; Wilson, M. A. J. Org. Chem. 2002, 67, 8469.

[43] Rasrendra, C. B.; Fachri, B. A.; Makertihartha, I. G. B. N.; Adisas mito, S.; Heeres, H. J. ChemSusChem 2011, 4, 768. 